\title{
Arsenicals in Ayurveda - A Review through Brihatrayi
}

\author{
Review Article
}

\author{
Shivanad Biradar ${ }^{1}$, Swapnil Y Chaudhari ${ }^{1}$, Galib R $^{2 *}$, Prajapati PK ${ }^{3}$ \\ 1. PhD Scholar, 2. Assistant Professor, 3. Professor \\ Department of Rasa Shastra and Bhasihajya Kalpana including Drug Research \\ Institute for Post Graduate Teaching and Research in Ayurveda \\ Gujarat Ayurved University, Jamnagar
}

\begin{abstract}
Metals and minerals including heavy metals like mercury, lead and arsenic are most frequently utilized in Ayurvedic system of medicine. Use of arsenicals is an integral component in Ayurvedic therapeutics. In Ayurveda, arsenicals comprise Haratala (Arsenic trisulphide), Manahshila (Arsenic disulphide) and Somala (Arsenic trioxide). They were widely practiced for various therapeutic and non-therapeutic purposes by the seers in different pathological manifestations with great conviction. Classics of Brihatrayi, one of the scheduled books of Ayurveda also hold ample references of arsenicals in diseases like Hicca (hiccup), Shwasa (respiratory distress), Shotha (oedema), Visha (Poison) and Kushta (skin diseases) etc. In the current review, an attempt has been made to compile all such references of arsenicals referred in Brihatrayi.
\end{abstract}

Key Words: Arsenic, Ayurveda, Brihatrayi, Haratala, Manahshila, Somala

\section{Introduction:}

India is having glorious history of rich traditional medical practices. Ayurveda is one among widely practiced ancient medical system in Indian subcontinent dates back to about 5000 years BC. It comprises utilization of drugs originated from natural resources including metals and minerals to maintain or cure or prevent different diseases. These formulations are being used successfully without any noticeable side effects. Seers have experienced the usefulness of metals and minerals and documented them in their respective classics. Being used for

*Corresponding Author:

\section{Galib R,}

Assistant Professor,

Department of rasaShastra and Bhaisajya

Kalpana including Drug Research,

IPGTR \& A, GAU, Jamnagar

Email id-galib14@yahoo.co.in over a long period, these medicines are acknowledged to be safe, which is the ultimate proof for their non - toxic beneficial effects. But there is big hue and cry about presence of heavy metals in Ayurvedic drugs about their safety aspects. Few toxicity reports were published due to administration of Ayurvedic drugs. $(1,2,3)$ Toxicity may be noticed due to noncompliance of code of conduct of the treatment procedures. Seers were also aware about such toxic effects. They brilliantly provided certain precautions and contraindications while manufacturing and using metals and minerals. Great care has been imposed while using all such metallic preparations in therapeutics.(4)

Arsenicals, a group of minerals known to human since antiquity comprising Haratala (Arsenic trisulphide), Manahshila (Arsenic disulphide) and Somala (Arsenic trioxide) mentioned with wide range of therapeutics. Though 
therapeutic utility of arsenicals was increased with advent of Rasashastra, references pertaining to arsenicals can be traced back in major treatises too. But, this information is scattered in the classics. Though, one attempt has been reported on compiling references of metals and minerals; it was limited only to Charaka Samhita. $(5,6)$ Considering this, references of arsenicals available in Brihatrayi are compiled and grouped into categories with an intension to provide emphasis on therapeutic and non-therapeutic usage of them. The categories are as under:

Utilization in therapeutics Internal administration External application

Other purposes etc.

\section{Materials and Methods:}

Brihatrayi (Caraka Samhita, Sushruta Samhita and Astanga Hridaya) with their commentaries are screened to compile references of arsenicals.

\section{Results and Observations: \\ Arsenicals in Brihatrayi:}

Haratala, Manahshila and Somala are the three elements that contain Arsenic as an element in their composition mentioned in Brihatrayi.

\section{Classification:}

Both Charaka and Sushruta have grouped Haratala and Manahshila under Parthiva Dravyas, while, Sushruta categorized Haratala and Somala under Dhatu Visha.

\section{Synonyms:}

Charaka used two terms i.e. Alam, Haratala to refer Haratala. Chakrapani clarified that both these terms are synonymously used. Sushruta used terms Ala, Tala and Harita to refer this mineral.

Charaka used only one term i.e. Manahshila throughout the classic, while
Sushruta mentioned Nepali, Manohva and Shila to refer Manahshila. Vagbhata used one more term Manohva for Manahshila.

Sushruta used the term Phenashma for Somala.

\section{Haratala:}

Chemically Haratala is identified as Orpiment $\left(\mathrm{As}_{2} \mathrm{~S}_{3}\right)$. Being an arsenical mineral; it should be administered with great caution.(7) It is advocated externally as well as internally since the period of Charaka Samhita. It is used as a component in formulations of topical application like Lepa, Taila, Anjana etc. Charaka recommended its formulations for internal administration to treat diseases like Hikka (hiccup), Shvasa (respiratory distress), Kasa (cough) and Visha (poisoning). The therapeutic dose mentioned for Haritala Bhasma is $30-60$ mg.(8) The formulations mentioned in Brihatrayi, which hold Haratala as one of the components are depicted at Table 1, 2 and 3 .

\section{Manahshila:}

Chemically, Manahshila is identified as Realgar - Red Orpiment $\left(\mathrm{As}_{2} \mathrm{~S}_{2}\right)$.(9) It is extensively used in formulating compounds for internal administration and topical application by ancient seers. It is advocated for internal administration in various dosage forms to treat diseases like Kasa, Hikka, Swasa, Chhardi, Visha and Pinasa. Ample of formulations containing Manahshila as an integral component were prescribed for external applications to treat different skin diseases, eye diseases etc. Charaka and Vagbhata used Manahshila in anti-dotal formulations for snake bite and scorpion sting. This mineral is frequently preferred by the seer for administration through different routes of drug administration. The therapeutic dose mentioned for Manahshila is 4-8 mg.(10) The formulations mentioned in Brihatrayi, which hold Manahshila as one of the 
components, are summarized at Table 4, 5, 6.

Formulations containing both Haratala and Manahshila as components in Sushruta Samhita and Astanga Hridaya are depicted at Table 7, 8 .

\section{Somala:}

No reference pertaining to Somala is available in Charaka Samhita and Astanga Hridaya. Sushruta used the term Phenashma to refer this mineral and grouped it under Dhatu Visha.

\section{Discussion:}

Screening of classics of Brihatrayi revealed that arsenicals were used to treat many pathological manifestations since ages. Though, the use of metals and minerals in therapeutics was increased after the development of Rasashastra but roots of this science can be traced back to Brihatrayi. They categorized Haratala and Manahshila under the group of Parthiva Dravya. Sushruta classified Haratala and Phenashma under Sthavara (Dhatu) Visha. Charaka advocated formulations of Haratala and Manahshila in certain pathological lesions involving skin.

Arsenicals are used in different pharmaceutical dosage forms like Lepa, Dhooma, Anjana, Churna and Taila etc. Formulations of arsenicals are recommended in various diseases of skin, eye, oral cavity and respiratory tract etc. This information available in classics makes clear that such metals have special role in different clinical conditions. They have made their unique place in Ayurvedic pharmacopoeia and became integral part of Ayurvedic therapeutics. Formulations of arsenicals are safely used in Ayurvedic therapeutics. Various published reports revealed that when these minerals are converted into medicines strictly adhering to the classical guidelines specified in ancient texts, they are devoid of any toxicity.(11,12) Sameer Pannaga Rasa, one among arsenical formulation has a significant action in cases bronchial asthma and it could suppress eosinophil count, Erythrocyte Sedimentation Rate and can improve Peak Expiratory Flow Rate (PEFR) along with providing symptomatic relief.(13)

Current research work clearly reflects that arsenicals are safely used in Ayurveda in different dosage form to treat various disease conditions. These classical evidences are ultimate proof for their safety as they are clinically tested since ages without any noticeable side effects. So that mere presence of heavy metals in Ayurvedic formulations is not sufficient to say that Ayurvedic drugs are toxic.

\section{Conclusion:}

Screening of Brihatrayi reveals that arsenicals have significant therapeutic values. Fine powders of these processed minerals are used in therapeutics through both internal and external routes. Majority of the formulations are for external application. Certain Dhuma Yogas also contain Haratala that have been advocated in diseases of respiratory tract. Being used for over a long period, these medicines are acknowledged as safe, which is the ultimate proof for their non toxic beneficial effects. But taking consideration about potential toxicity of arsenicals; precautions should be taken to avoid any harmful effects resulting from their use in therapeutics. Well designed pre-clinical and clinical studies also can be planned to evaluate actual safety and therapeutic efficacies in respective conditions. This work is anticipated to be convenient for referring the utilization of arsenicals emphasized in Brihatrayi and reduces the sternous efforts of researchers to gather information. This also emphasizes the frequency of arsenicals used in different conditions since ancient days of civilization.

\section{References:}

1 Sathe K, Ali U, Ohri A. Acute renal failure secondary to ingestion of 
Ayurvedic medicine containing mercury. Indian Journal of Nephrology 2013; 23(4): 301-303.

2 Saper R, Kales S, Paquin J, Burns M, Eisenberg D, Davis R et al. Heavy Metal Content of Ayurvedic Herbal Medicine Products. JAMA 2004; 292: 2868-73.

3 Kales S, Christophi C and Saper R. Hematopoietic toxicity from leadcontaining Ayurvedic Medications. Med Sci Monit 2007; 13(7): CR295CR298.

4 Agnivesha, Charaka Samhita, Ayurveda Dipika Commentary by Chakrapanidutta, Chikitsa 12/21-2, Chaukhambha Surbharati Prakashan, Varanasi, Reprint 2000.

5 Galib, Metals and Minerals in Ancient Indian Literature - A Review through Charaka Samhita. Chowkhamba Krishnadas Academy, Varanasi, $1^{\text {st }}$ Ed., 2013.

6 Galib, Mayur B, Mayur M, Chandrashekhar J, Patgiri BJ, Prajapati $\mathrm{PK}$, Therapeutic potentials of metals in ancient India: A review through Charaka Samhita, Journal of Ayurveda and Integrative Medicine, April - June 2011, Vol 2, issue 2. 55 - 63

7 Ayurvediya Rasashastra. Jha CB, Chaukhamba Surabharati Prakashan, Reprint Ed., Varanasi, pp 257 (2003).
8 Rasatarangini. Prasadani Commentary by Haridutta Shastri, Ed. Kashinath Shastri, Motilal Banarasidas, New Delhi, 11th Ed., Reprint, 11/ 56, pp 253 (2000).

9 Ayurvediya Rasashastra. Jha CB, Chaukhamba Surabharati Prakashan, Varanasi, pp 263 (2003).

10 Rasatarangini. Prasadani Commentary by Haridutta Shastri, Ed. Kashinath Shastri, Motilal Banarasidas, New Delhi, 11th Ed., Reprint, 11/ 117, pp 263 (2000).

11 Sud S, Reddy P, Sujatha $\mathrm{K}$ and Honwad S. A comparative toxicological study of Rasamanikya prepared with three different methods. Ayu 2013; 34(3): 309-315.

12 Begum G, Hamid K and Choudhuri M. Changes in lipid profile of rat plasma after chronic administration of Mallasindura (MSL) - an Ayurvedic metallic preparation. J. Pharm. Sci. \& Res 2015; 7(3): 123-126.

13 Mashru M, Galib R, Shukla V, Ravishankar B, Prajapati P. Effect of Sameera Pannaga Rasa (arsenomercurial formulation) in the management of Tamaka Shwasa (bronchial asthma) - Randomized double blind clinical study. AYU 2013; 34(4): 346-351. 
Table 1: Therapeutic utilization of Haratala in Charaka Samhita

\begin{tabular}{|c|c|c|c|}
\hline & Reference & Formulation & Therapeutic Uses \\
\hline \multicolumn{4}{|c|}{ Utilization in therapeutics : Internal Administration } \\
\hline 1 & Sutra 5/26 & Dhooma Yoga & Shiro Virechana \\
\hline 2 & Chikitsa $17 / 78$ & Dhooma Yoga & Hikka, Swasa \\
\hline 3 & Chikitsa 18/69 & Dhooma Yoga & Vataja Kasa \\
\hline 4 & Chikitsa $18 / 74$ & Dhooma Yoga & Vataja Kasa \\
\hline 5 & Chikitsa 23/54 & Mrit Sanjivani Agada & Sarva Visha \\
\hline \multicolumn{4}{|c|}{ Utilization in therapeutics : External Application } \\
\hline 6 & Sutra $3 / 5$ & Pradeha Yoga & Kushtha \\
\hline 7 & Sutra $3 / 10$ & Avacurna Yoga & Kushtha \\
\hline 8 & Sutra 3/12 & Pradeha Yoga & Kushtha \\
\hline 9 & Chikitsa 7/114 & Kanaka Kshiri Taila & Krimi, Kandu, Kushtha \\
\hline $\mathbf{1 0}$ & Chikitsa 9/66 & Varti Yoga & Apasmara, Unmada \\
\hline 11 & Chikitsa 14/56 & Lepa Yoga & Arsha \\
\hline 12 & Chikitsa 25/114 & Lepa Yoga & Twak Janana \\
\hline 13 & Chikitsa 26/196 & Peetaka Churna & Mukha Roga \\
\hline \multicolumn{4}{|c|}{ Other References } \\
\hline 14 & Sutra $1 / 70$ & Referred as 'Alam' & $\begin{array}{l}\text { Classification of Parthiva } \\
\text { Dravya }\end{array}$ \\
\hline
\end{tabular}

Table 2: Therapeutic utilization of Haratala in Sushruta Samhita

\begin{tabular}{|c|l|l|l|}
\hline $\begin{array}{c}\text { Sr } \\
\text { No }\end{array}$ & \multicolumn{1}{|c|}{ Reference } & \multicolumn{1}{c|}{ Formulation } & \multicolumn{1}{|c|}{ Therapeutic Uses } \\
\hline Utilization in therapeutics : External Application \\
\hline $\mathbf{1}$ & Sushruta Chikitsa 1/105 & Romashatana karma & Vrana \\
\hline $\mathbf{2}$ & Sushruta Chikitsa 1/107 & Romashatana karma & Vrana \\
\hline $\mathbf{3}$ & Sushruta Chikitsa 1/108 & Romashatana karma & Vrana \\
\hline $\mathbf{4}$ & Sushruta Chikitsa 6/12 & Taila Lepa & Arsha \\
\hline $\mathbf{5}$ & Sushruta Chikitsa 9/27 & Lepa & Shwitra \\
\hline $\mathbf{6}$ & Sushruta Chikitsa 18/19 & Taila & Granthi, Arbuda \\
\hline $\mathbf{7}$ & Sushruta Chikitsa 20/28 & Lepa & Arunshika \\
\hline $\mathbf{8}$ & Sushruta Chikitsa 20/59 & Lepa & Ahiputana, Mushka Kacchu \\
\hline $\mathbf{9}$ & Sushruta Uttara 21/52 & Ear drops with cow urine & Krumikarna \\
\hline $\mathbf{1 0}$ & Sushruta kalpa 6/3-7 & Ksharagada & Visha \\
\hline Other References & $\begin{array}{l}\text { Haratala and Somala } \\
\text { listed as Sthavara Visha }\end{array}$ & Dhatu visha \\
\hline $\mathbf{1 1}$ & Kalpa 2/5 &
\end{tabular}

Table 3: Therapeutic utilization of Haratala in Ashtanga Hridaya

\begin{tabular}{|c|l|l|l|}
\hline Sr. No & Reference & Formulation & Therapeutic uses \\
\hline Utilization in therapeutics : External Application & \\
\hline 1 & Chikitsa 20/13 & Lepa & Shwitra \\
\hline 2 & Uttara 13/31 & Bhaskaranjana II & Timira \\
\hline 3 & Uttara 14/30 & Varti & Based on Upadrava \\
\hline 4 & Uttara 16/56 & To grow eyelashes & Pilla Roga for Romotpatti \\
\hline 5 & Uttara 37/35 & Lepa \& Anjana & Scorpion bite \\
\hline 6 & Uttara 37/40 & Lepa \& Anjana & Scorpion bite \\
\hline
\end{tabular}


Table 4: Therapeutic utilization of Manahshila in Charaka

\begin{tabular}{|c|c|c|c|}
\hline & Reference & Formulation & Therapeutic Uses \\
\hline \multicolumn{4}{|c|}{ Utilization in therapeutics : Internal Administration } \\
\hline 1 & Sutra 5/26 & Dhooma Yoga & Shiro Virechana \\
\hline 2 & Chikitsa 17/77 & Dhooma Yoga & Hikka, Swasa \\
\hline 3 & Chikitsa $17 / 145$ & Manahshiladi Ghrita & Hikka, Swasa \\
\hline 4 & Chikitsa $18 / 52$ & Leha Yoga & Kasa, Hikka, Swasa \\
\hline 5 & Chikitsa 18/69 & Dhooma Yoga & Vataja Kasa \\
\hline 6 & Chikitsa 18/71 & Dhooma Yoga & Vataja Kasa \\
\hline 7 & Chikitsa 18/73 & Dhooma Yoga & Vataja Kasa \\
\hline 8 & Chikitsa 18/74 & Dhooma Yoga & Vataja Kasa \\
\hline 9 & Chikitsa $18 / 75$ & Dhooma Yoga & Vataja Kasa \\
\hline 10 & Chikitsa 18/130 & Dhooma Yoga & Vataja Kasa \\
\hline 11 & Chikitsa 18/146 & Dhooma Yoga & Ksataja Kasa \\
\hline 12 & Chikitsa 18/147 & Dhooma Yoga & Ksataja Kasa \\
\hline 13 & Chikitsa 18/169 & Haritaki Leha & Swasa, Kasa \\
\hline 14 & Chikitsa 20/39 & Leha Yoga & Chardi \\
\hline 15 & Chikitsa 23/55 & Mrita Sanjivani Agada & Visha \\
\hline 16 & Chikitsa 26/152 & Pradhamana Nasya Yoga & Pinasa \\
\hline \multicolumn{4}{|c|}{ Utilization in therapeutics : External Application } \\
\hline 17 & Sutra $3 / 5$ & Pradeha Yoga & Kushtha \\
\hline 18 & Sutra $3 / 10$ & Avacurna Yoga & Kushtha \\
\hline 19 & Sutra 3/12 & Pradeha Yoga & Kushtha \\
\hline 20 & Sutra $3 / 12$ & Pradeha Yoga & Kushtha \\
\hline 21 & Sutra $3 / 15$ & Lepa Yoga & Kushtha \\
\hline 22 & Chikitsa 3/306 & Anjana Yoga & Vishama Jwara \\
\hline 23 & Chikitsa 7/117 & Lepa Yoga & Sidhma \\
\hline 24 & Chikitsa 7/167 & Shamana Lepa & Switra \\
\hline 25 & Chikitsa $7 / 170$ & Lepa Yoga & Kilasa \\
\hline 26 & Chikitsa 23/78 & Agadahara Lepa Yoga & Visha, Timira etc. \\
\hline 27 & Chikitsa 23/190 & Lepa Yoga & Sarva Shotha, Visha \\
\hline 28 & Chikitsa 23/192 & Lepa Yoga & Sarva Visha \\
\hline 29 & Chikitsa 23/213 & Pancha Shirisha Agada & Vishahara \\
\hline 30 & Chikitsa 25/114 & Lepa Yoga & Twak Janana \\
\hline 31 & Chikitsa 26/196 & Peetaka Churna & Mukha Roga \\
\hline 32 & Chikitsa 26/235 & Varti Yoga & Netra Roga \\
\hline 33 & Chikitsa 26/250 & Anjana Yoga & Netra Roga \\
\hline 34 & Chikitsa 26/252 & Sukhavati Varti & Netra Roga \\
\hline \multicolumn{4}{|c|}{ Other References } \\
\hline 35 & Sutra $1 / 70$ & \multicolumn{2}{|c|}{ Classified under Parthiva Dravya } \\
\hline
\end{tabular}

Table 5: Therapeutic utilization of Manahshila in Sushruta Samhita

\begin{tabular}{|c|l|l|l|}
\hline Sr.No & Reference & Formulation & Therapeutic uses \\
\hline Utilization in therapeutics : Internal Administration \\
\hline 1. & Chikitsa 40/4 & Dhooma varti & Kasa \\
\hline 2. & Uttara 51/50 & Dhooma varti & Hikka, Shwasa \\
\hline
\end{tabular}




\section{Utilization in therapeutics : External Application}

\begin{tabular}{|c|l|l|l|}
\hline 3. & Chikitsa 2/69 & Ropana Taila & Vrana Chikitsa \\
\hline 4. & Chikitsa 20/24 & Lepa & Indralupta \\
\hline 5. & Chikitsa 20/54 & Ropana Taila & Valmika \\
\hline 6. & Uttara 11/16 & Anjana & Kandu \\
\hline 7. & Uttara 12/16 & Anjana & Sirotpata \\
\hline 8. & Uttara 12/29 & Anjana & Shukra nashaka \\
\hline 9. & Uttara 13/7 & Application & Lekhyaroga \\
\hline 10. & Uttara 14/4 & Application & Bhedhyaroga (Bisgranthi) \\
\hline 11. & Uttara 14/7 & Application & Bhedhyaroga Anjananamika) \\
\hline 12. & Uttara 17/7 & Anjana & Drushtigata roga \\
\hline 13. & Uttara 17/18 & Varti & Ratryandha \\
\hline 14. & Uttara 17/39 & Rasakriya & Pittaja Timira \\
\hline 15. & Uttara 17/43 & Rasakriya & Kaphaja Timira \\
\hline 16. & Uttara 18/98 & Anjana & Netraroga \\
\hline 17. & Uttara 18/100 & Anjana & Timira \\
\hline 18. & Uttara 19/14 & Anjana & Nayana Abhighata \\
\hline 19. & Uttara 21/48 & Taila & Karna Roga \\
\hline 20. & Uttara 26/34 & Nasya & Shiroroga \\
\hline 21. & Uttara 35/7 & Bali in Goshala & Mukhamandika \\
\hline 22. & Uttara 39/263 & Nasya & Jwara Pratishedha \\
\hline 23. & Uttara 50/18 & Swedana & Hikka \\
\hline Other References & Parthiva Drug & Classification \\
\hline 24. & Sutra 1/32 & \multicolumn{2}{|l|}{} \\
\hline
\end{tabular}

Table 6: Therapeutic utilization of Manahshila in Ashtanga Hridaya

\begin{tabular}{|c|c|c|c|}
\hline Sr. No. & Reference & Formulation & Therapeutic uses \\
\hline \multicolumn{4}{|c|}{ Utilization in therapeutics : Internal Application } \\
\hline 1 & Chikitsa 3/168 & Haritaki Avaleha & Swasa, Kasa \\
\hline 2 & Chikitsa 6/20 & Licking with honey & Chardi, Trishna, Hridroga \\
\hline \multicolumn{4}{|c|}{ Utilization in therapeutics : External Application } \\
\hline 3 & Chikitsa $1 / 162$ & Anjana & Vishama jwara \\
\hline 4 & Chikitsa $13 / 38$ & Taila nirmana & Medoja Vriddhi \\
\hline 5 & Chikitsa 19/73 & Lepa & Sidhma and Switra \\
\hline 6 & Uttara $9 / 23$ & Pratisarana & Kaphotklishtha roga \\
\hline 7 & Uttara $11 / 24$ & Anjana & Timira, Arma \\
\hline 8 & Uttara $13 / 25$ & Anjana & Timira \\
\hline 9 & Uttara $13 / 29$ & Bhaskaranjana & Timira \\
\hline 10 & Uttara $13 / 70$ & Vimala Varti & Timira \\
\hline 11 & Uttara $16 / 5$ & Lepa & Kaphaja Abhishyanda \\
\hline 12 & Uttara $16 / 5$ & Pottali & Netraroga \\
\hline 13 & Uttara $16 / 52$ & Anjana & Kandu \\
\hline 14 & Uttara $24 / 28$ & Lepa & Indralupta \\
\hline 15 & Uttara $36 / 82$ & Vajragada & Snake poison \\
\hline 16 & Uttara $37 / 44$ & Antidote & Scorpion poison \\
\hline
\end{tabular}


Table 7: Therapeutic utilization of Haratala and Manahshila in Sushruta Samhita

\begin{tabular}{|c|c|c|c|}
\hline Sr.No. & Reference & Formulation & Therapeutic uses \\
\hline \multicolumn{4}{|c|}{ Utilization in therapeutics : Internal Application } \\
\hline 1. & Uttara $52 / 22$ & Dhooma varti & Kasa \\
\hline \multicolumn{4}{|c|}{ Utilization in therapeutics : External Application } \\
\hline 2. & Sutra $37 / 14$ & Varti & Samshodhana in Nadivrana \\
\hline 3. & Sutra $37 / 18$ & Taila & Samshodhana \\
\hline 4. & Chikitsa $1 / 60$ & Lepa & Vrana \\
\hline 5. & Chikitsa 1/97 & Shwetakarma & Vrana \\
\hline 6. & Chikitsa 2/91 & Taila & Dushta Vrana \\
\hline 7. & Chikitsa 9/10 & Lepa & Kushtha \\
\hline 8. & Chikitsa 9/55 & Vajraka Taila & Kushtha, Vrana \\
\hline 9. & Chikitsa 18/41 & Rubbing & Vrana \\
\hline 10. & Chikitsa 19/18 & Taila LA & Medoja Vriddhi \\
\hline 11. & Chikitsa 19/40 & Churna for LA & Upadansha \\
\hline 12. & Chikitsa 19/46 & Churna & Upadansha, Visarpa \\
\hline 13. & Chikitsa 20/6 & Kalka lepa & Kshudra roga \\
\hline 14. & Chikitsa 20/21-23 & Lepa & Alasa roga \\
\hline 15. & Chikitsa 25/38 & Ghrita Abhyanga & Vyanga \\
\hline 16. & Kalpa 8/112 & With honey & Mutra Visha Dansha Chi. \\
\hline 17. & Uttara $11 / 9$ & Anjana, Varti & Kaphaja Netrabhishyanda \\
\hline 18. & Uttara 30/7 & with Karanja & Shakuni Pratishedha \\
\hline 19. & Uttara $32 / 4$ & Taila & Putana Pratishedha \\
\hline 20. & Uttara $33 / 3$ & Taila & Andhaputana \\
\hline 21. & Uttara 60/49 & Taila, ghrita & Upasarga \\
\hline
\end{tabular}

Table 8: Therapeutic utilization of Haratala \& Manahshila in Ashtanga Hridaya

\begin{tabular}{|c|c|c|c|}
\hline Sr.No. & Reference & Formulation & Therapeutic uses \\
\hline \multicolumn{4}{|c|}{ Utilization in therapeutics : Internal Application } \\
\hline 1. & Chikitsa 3/68 & Dhoomapana & Kapha-Vataja Kasa \\
\hline 2. & Sutra 30/18 & Ksharakarma & Kshara \\
\hline 3. & Chikitsa 4/11 & Dhoomapana & Swasa \\
\hline 4. & Chikitsa 3/149 & Dhoomapana & Kasa \\
\hline 5. & Chikitsa 3/150 & Dhoomapana & Kasa \\
\hline 6. & Uttara 21/18 & Dhooma & Shirovirechana \\
\hline \multicolumn{4}{|c|}{ Utilization in therapeutics : External Application } \\
\hline 7. & Chikitsa.19/67,70,71 & Churna (LA) & Dadru \\
\hline 8. & Chikitsa 19/81 & Maha Vajraka Taila & Switra, Arsha LA \\
\hline 9. & Uttara $1 / 23$ & Lepa & $\begin{array}{l}\text { Balaka Namakaran } \\
\text { Samskara }\end{array}$ \\
\hline 10. & Uttara $2 / 73$ & Lepa & Ahiputana Chikitsa \\
\hline 11. & Uttara $5 / 2$ & Internal \& external & $\begin{array}{l}\text { Graha Unmada, } \\
\text { Apasmara }\end{array}$ \\
\hline 12. & Uttara $13 / 87$ & Varti & Ratryandha \\
\hline 13. & Uttara $20 / 24$ & Varti & Putinasa \\
\hline 14. & Uttara 22/100 & Peetaka Churna & $\begin{array}{l}\text { Mukha Roga (hold in } \\
\text { mouth) }\end{array}$ \\
\hline 15. & Uttara 25/49 & Lepa & Vrana \\
\hline
\end{tabular}


International Journal of Ayurvedic Medicine, 2015, 6 (2), 113-121

\begin{tabular}{|c|l|l|l|}
\hline 16. & Uttara 25/60 & Lepa & Twak Shuddhi \\
\hline 17. & Uttara 30/20 & Taila pana & Nadivrana Chi. \\
\hline 18. & Uttara 32/2 & Lepa & Yavaprakhya \\
\hline 19. & Uttara 32/31 & Manjishthadi taila & Vyanga, Nilika \\
\hline 20. & Uttara 34/4 & Lepa & Guhya roga \\
\hline 21. & Uttara 35/24 & Chandrodaya Agada & Poison \\
\hline 22. & Uttara 37/73 & Mandara Agada & Poison \\
\hline
\end{tabular}

